\title{
Manejo perioperatorio de nuevos fármacos psiquiátricos
}

\section{Perioperative management of new psychiatric drugs}

\author{
Maximiliano Zamora H. ${ }^{1}$, Constanza Lema H. ${ }^{2}$
}

\begin{abstract}
Psychoactive drugs, including antidepressants and antipsychotics, are currently one of the most commonly used drugs, so we often find patients who consume them during the perioperative period. Historically, they have been associated with multiple and serious adverse effects, such as serotonin syndrome, but nowadays these are infrequent, especially due to the good safety profile of the new drugs most commonly used. Therefore, it is recommended to keep these drugs in the perioperative period, to avoid adverse effects related to their suspension. Among the novel and most used antidepressants are the socalled duals, such as venlafaxine, desvenlafaxine and duloxetine, these are safe and it is recommended to maintain their use. The same is recommended with drugs such as trazodone, bupropion and mirtazapine. Another antidepressant, vortioxetine, has not reported significant adverse effects in the perioperative period, so it is recommended to maintain its use. Agomelatine, derived from melatonin, is considered safe to maintain and could have beneficial effects by reducing preoperative anxiety and eventually reducing the incidence of postoperative delirium in susceptible patients. Antipsychotics are safe in the perioperative period and, in general, it is recommended to maintain their use.
\end{abstract}

\section{RESUMEN}

Los fármacos psiquiátricos, entre los que se encuentran los antidepresivos y antipsicóticos, son de los fármacos más utilizados en la actualidad, por lo que

\section{Key words:}

Anesthetics, adverse effects, drug interactions, Serotonin syndrome, Antidepressive agents, adverse effects

\section{Palabras clave:}

Anestésicos, efectos adversos, interacción de drogas, síndrome serotoninérgico, antidepresivos, efectos adversos

1 Anestesiólogo, Residente de Anestesiología Cardiovascular. División de Anestesiología. Escuela de Medicina, Facultad de Medicina. Pontificia Universidad Católica de Chile.

2 Interna de Medicina. Escuela de Medicina, Facultad de Medicina. Universidad Austral de Chile.

Fecha de recepción: 14 de enero de 2019

Fecha de aceptación: 28 de febrero de 2019

\section{ORCID}

https://orcid.org/0000-0003-4895-7349

Fuente de financiamiento: Los autores no recibieron patrocinio para llevar a cabo este artículo.

Conflicto de intereses: Los autores declaran no tener conflicto de intereses.

\section{Correspondencia:}

Maximiliano Zamora $\mathrm{H}$.

Email:mszamora@uc.cl 
con frecuencia nos encontramos con pacientes que los consumen en el perioperatorio. Históricamente se han relacionado con múltiples y graves efectos adversos, como el síndrome serotoninérgico, pero hoy en día estos son infrecuentes, sobre todo por el buen perfil de seguridad que presentan los nuevos fármacos más utilizados. Por lo anterior, es que en general se recomienda mantener estas drogas en el perioperatorio, para evitar efectos adversos relacionados con su suspensión. Entre los antidepresivos más utilizados se encuentran los denominados duales, como venlafaxina, desvenlafaxina y duloxetina, estos son seguros y se recomienda mantener su uso. Lo mismo se recomienda con drogas como trazodona, bupropión y mirtazapina. Otro más novedoso, la vortioxetina, hasta el día de hoy no ha reportado efectos adversos relevantes en el perioperatorio, por lo que se recomienda mantener su uso. La agomelatina, derivada de la melatonina, se considera segura de mantener y podrían tener efectos beneficiosos al reducir la ansiedad preoperatoria y eventualmente reducir la incidencia de delirium postoperatorio en los pacientes susceptibles. Los antipsicóticos son seguros en el perioperatorio y en general se recomienda mantener su uso.

\section{Introducción}

A ctualmente, en nuestro país, los fármacos para distintos trastornos psiquiátricos, como antidepresivos, estabilizadores del ánimo, antipsicóticos, entre otros, son de los que se utilizan con mayor frecuencia, ya que en conjunto estas enfermedades afectan a una gran proporción de la población general. De hecho, se sabe que en chile su consumo diario aumentó en más de cuatro veces, desde los años 1992 a 2004 (11,7 personas por cada 1.000 habitantes)[1]. En Estados Unidos, los antidepresivos se encuentran entre los 3 grupos de fármacos que más se prescriben, en conjunto con los analgésicos e hipoglicemiantes[2].

Este tipo de pacientes tiene un riesgo elevado de complicaciones en el perioperatorio[3],[4], por lo que el anestesiólogo no solo debe estar consciente de la presencia de estas patologías, sino que del manejo perioperatorio de sus posibles complicaciones y eventuales efectos adversos que se podría relacionar a los fármacos utilizados. Además de lo anterior, el panorama se ve complejizado por el creciente número de fármacos existentes, lo que muchas veces dificulta el manejo perioperatorio[5].

Si bien existen revisiones con respecto al tema del manejo de este tipo de medicamentos en el perioperatorio, en la presente revisión abordaremos solo los nuevos fármacos psiquiátricos que han aparecido en el mercado en el último tiempo, con el fin de poder aclarar dudas en relación a su uso.

\section{Los problemas clásicos de los fármacos psiquiátricos en el perioperatorio}

El primer concepto relevante de entender, es que la gran mayoría de los fármacos psiquiátricos que se utilizan hoy en día, son muy seguros en el perioperatorio. Cada vez se cuentan con drogas con menos efectos adversos y un perfil de seguridad mayor.

A pesar de lo anterior, estas drogas sí pueden presentar efectos adversos relevantes e incluso graves, además de algunas interacciones farmacológicas. Lo anterior es lo que, históricamente, ha generado gran duda en cuanto a su manejo perioperatorio. Un efecto adverso que con frecuencia se menciona, relacionado al uso de antidepresivos que aumentan la disponibilidad de serotonina, es el síndrome serotoninérgico. Esto es provocado por un aumento excesivo en los niveles de serotonina en el sistema nervioso central, que puede ocurrir con dosis terapéuticas de estas drogas, pero con mayor frecuencia se asocia a sobredosis o interacciones de drogas que determinan sinergismo en la actividad serotoninérgica[6],[7]. Este se manifiesta con la tríada de inestabilidad autonómica, alteraciones neuromusculares y cambios en el estado mental del paciente. Su incidencia el día de hoy es bastante baja, e inicialmente los casos reportados se asociaron en mayor medida al uso de inhibidores de la monoaminooxidasa (IMAO), un grupo de antidepresivos, que, si bien hasta el día de hoy se utiliza para casos en que hay resistencia al tratamiento antidepresivo de primera línea, se utiliza con mucho menor frecuencia, 
comparándolos con los inhibidores selectivos de la recaptura de serotonina y los antidepresivos duales.

Los IMAO también presentan con frecuencia interacción con drogas utilizadas en el perioperatorio, como opioides (entre estos se han descrito casos relacionados a tramadol y fentanyl[6]), donde aumentaría el riesgo de presentar síndrome serotoninérgico si se utilizan estas drogas en forma concomitante. Como ya mencionamos, el uso de estas drogas hoy es menos frecuente, pero si un paciente las tuviera entre sus fármacos en el perioperatorio, la recomendación en general es suspenderlas al menos 2 semanas previo a la cirugía.

Con frecuencia también se habla del riesgo de sangrado aumentado que tienen los pacientes en tratamiento con antidepresivos. Con estos hay una reducción en los niveles plaquetarios de serotonina, lo que potencia el efecto sobre las mismas de los antiinflamatorios no esteroidales y aumenta el riesgo de sangrado en pacientes usuarios de Warfarina y los nuevos anticoagulantes orales directos[8]. Si bien no es el objetivo de esta revisión revisar el manejo de este conjunto de drogas en el perioperatorio, se debe tener en cuanta este riesgo aumentado de sangrado, sobretodo cuando se utilizan varias de estas drogas de forma simultánea. Si es relevante mencionar, que esto no contraindica el uso de las técnicas neuroaxiales en pacientes usuarios de antidepresivos, pero si es necesario evaluar caso a caso el perfil de coagulación de estos pacientes.

Otros efectos adversos que se mencionan con frecuencia, son taquicardia y aumento de las cifras de presión arterial, sobretodo cuando los antidepresivos tienen como mecanismo de acción el aumento de noradrenalina. Este efecto adverso, que se presentaba con mayor frecuencia con los antidepresivos tricíclicos, hoy es menos frecuente, aunque todavía se ve con cierta frecuencia con algunos antidepresivos duales.

Si bien, como mencionamos, existen de forma histórica muchos efectos adversos relacionados con estas drogas, hoy, los más graves como el síndrome serotoninérgico y las interacciones de los IMAO, son mucho menos frecuentes. Las nuevas drogas cuentan cada vez con mayor seguridad, y mantenerlas pasa a ser lo fundamental para evitar efectos adversos.

\section{Nuevos antidepresivos}

Entre los fármacos antidepresivos, se encuentran múltiples familias. Los más utilizados son los inhibidores selectivos de la recaptura de serotonina (ISRS), que actúan, como menciona su nombre, inhibiendo la recaptura presináptica de este neurotransmisor. Entre los fármacos dentro de este grupo se encuentran la fluoxetina, sertralina, citalopram, escitalopram, entre otros. A pesar de que estos fármacos son de gran efectividad y por lo mismo, los más utilizados, hasta un 30\%[9] de pacientes con trastorno depresivo mayor pueden no responder a la terapia de primera línea y es en este contexto que se han desarrollado nuevas drogas para combatirlo.

Entre los fármacos de gran uso actualmente son los Ilamados antidepresivos duales, los cuales inhiben tanto la recaptura de serotonina, como de noradrenalina. En este grupo se encuentran la venlafaxina, duloxetina, desvenlafaxina[10]. La Venlafaxina tiene una vida media de 5 horas, mientras que su metabolito activo, la desvenlafaxina tiene una vida media de 11 horas. Duloxetina tiene una vida media de 12 horas. En cuanto a estos fármacos, entre algunos efectos adversos se encuentran la sedación, náuseas, insomnio, taquicardia, efectos anticolinérgicos y constipación[11]. Al igual que lo que podía ocurrir con los antidepresivos tricíclicos, al ser fármacos que aumentan la disponibilidad de noradrenalina, asociado a algunos agonistas adrenérgicos en el perioperatorio, se han descrito casos de aumentos relevantes de la presión arterial y la frecuencia cardíaca, pero los eventos en general son poco frecuentes y autolimitados. Venlafaxina y duloxetina son inhibidores del citocromo P450, específicamente CYP 2D6[7], los que podrían determinar alteraciones de la cinética de algunos fármacos, por otro lado, desvenlafaxina no cuenta con este efecto adverso. En suma, no se han reportado grandes efectos adversos de estas drogas en el perioperatorio, por lo que la recomendación general es mantenerlas. Sí se debe tener precaución con el uso de algunos agonistas adrenérgicos, que pueden gatillar episodios de taquicardia e hipotensión (como drogas vasoactivas, o incluso la ketamina).

La mirtazapina es un fármaco de acción dual serotoninérgica y noradrenérgica a través de los receptores alfa 2, que determina efectos antidepresivos, ansiólisis y sedantes. Su uso se considera seguro en el perioperatorio, por lo que también se recomienda su mantención[3], incluso, su uso preoperatorio se ha relacionado con una significativa reducción de la ansiedad, náuseas y vómitos postoperatorios[12]. La trazodona es un antagonista del receptor serotoninérgico 2A e inhibidor de la recaptura de serotonina, además, tiene efecto antihistamínico por lo que produce sedación, también se recomienda mantener en el perioperatorio[11]. El bupropión es un inhibidor 
de la recaptura de serotonina y dopamina, también se utiliza como antidepresivo, teniendo un efecto más activador, además de ser utilizado en el tratamiento de la deshabituación tabáquica. También se considera seguro mantenerlo en el perioperatorio.

La vortioxetina[13] es un antidepresivo mucho más novedoso, se define como multimodal ya que, además de actuar sobre los neurotransmisores monoaminérgicos habituales, también actúa sobre sistemas no monoaminérgicos (como el glutamato). Si bien no tienen una eficacia superior a los antidepresivos convencionales de primera línea, su novedoso mecanismo de acción daría algunos beneficios en los pacientes que presentan déficit cognitivos asociados a los trastornos depresivos[14]. Tiene una vida media de aproximadamente 66 horas. Los principales efectos adversos de esta droga son similares a los de otros antidepresivos, náuseas, vómitos y constipación. Al igual que los ISRS y el resto de los antidepresivos que alteran la recaptura de serotonina, tiene riesgo de síndrome serotoninérgico cuando se combinan con algunas drogas como los IMAO y un aumento del riesgo de sangrado. Hasta el momento, no hay grandes efectos adversos reportados relacionados al uso de esta droga en el perioperatorio, por lo que mantener su uso se considera seguro.

Una droga quizás mucho más común para nosotros, que en el último tiempo ha comenzado a ser utilizada con mayor frecuencia para el manejo de algunos trastornos depresivos, es la ketamina. Se utiliza en infusiones endovenosas con dosis bajas $(0,5$ $\mathrm{mg} / \mathrm{kg}$ ), generando un efecto antidepresivo que puede durar hasta 2 semanas, incluso se ha postulado que podría disminuir el riesgo inminente de suicidio y en general es un medicamento que se utiliza en depresiones resistentes a varios tipos de tratamientos[15],[16]. Como las dosis utilizadas son bajas, es poco probable que tenga efectos adversos para nosotros en el perioperatorio, pero sí se debe considerar reducir las dosis anestésicas, sobre todo si el paciente recibió su infusión poco antes de la cirugía.

Otro antidepresivo novedoso, por su mecanismo de acción ya conocido, es la agomelatina. Agonista sintético de la conocida melatonina, esta droga se utiliza para varios trastornos del sueño y ha demostrado también beneficios en algunos tipos de trastornos depresivos. Tiene una vida media de aproximadamente 2 horas[17]. Esta droga, al provocar ansiólisis y sedación, podría incluso servir como coadyuvante para la inducción anestésica. Incluso luego de la cirugía, este fármaco permite la restauración del sueño y prevención del delirium posoperatorio. Tiene un buen perfil de seguridad y sin efectos adversos reportados en el perioperatorio, por lo que se recomienda continuar su uso[18],[19].

\section{Antipsicóticos}

Los antipsicóticos se dividen en neurolépticos o antipsicóticos típicos (como el haloperidol) y antipsicóticos atípicos (como la risperidona y otros más nuevos como el aripiprazol)[20]. Los primeros presentan mayor incidencia de síntomas extrapiramidales como distonía, comparado con los antipsicóticos atípicos, que son los que se utilizan con mayor frecuencia en la actualidad.

Un efecto adverso muy poco frecuente, pero relevante de mencionar por su gravedad, es el síndrome neuroléptico maligno. Este se caracteriza por hipertermia, rigidez muscular y disfunción autonómica. Su importancia radica en que este es un efecto adverso que puede ocurrir en cualquier punto del tratamiento de estos pacientes $y$, además, es un importante diagnóstico diferencial cuando sospechamos una hipertermia maligna. El manejo es de soporte, pero se han utilizado dantrolene y relajantes neuromusculares para disminuir la rigidez.

Otro tema que con frecuencia se menciona, es la alteración del intervalo QT por este tipo de fármacos. Un intervalo QT alargado inducido por drogas se asocia con un riesgo aumentado de arritmias cardíacas y mortalidad cardiovascular. En general el riesgo es bajo, pero especialmente en el caso de los antipsicóticos atípicos, donde se ha demostrado específicamente que drogas como el aripiprazol y la olanzapina tienen un riesgo casi nulo[21].

Se recomienda mantener todos estos fármacos en el perioperatorio[3], ya que la suspensión aguda de estos fármacos puede precipitar episodios psicóticos agudos y su uso solo se ha asociado a una leve susceptibilidad a hipotensión relacionada con la anestesia general.

\section{Conclusiones}

Los fármacos psiquiátricos, entre los que destacamos los antidepresivos y antipsicóticos, se encuentran entre los más frecuentes utilizados por nuestros pacientes en el perioperatorio. Si bien existen muchos efectos adversos relevantes para el perioperatorio, hoy en día los más graves son infrecuentes, por lo que se recomienda, por lo general, mantener estos fármacos al momento de la cirugía. Entre los antidepresivos más nuevos y de mayor uso actual, se en- 
cuentran los antidepresivos duales y otros como la mirtazapina, bupropión, trazodona y vortioxetina, los que se consideran seguros y se recomienda mantener su uso. Los derivados de la melatonina como agomelatina, también se consideran seguros y por lo mismo se deben mantener, incluso podrían tener un efecto beneficioso al reducir la ansiedad previo a la cirugía y el delirium postoperatorio. Los antipsicóticos son fármacos seguros en el perioperatorio y se recomienda su mantención para evitar inicio de nuevos cuadros psicóticos. Es importante recordar al síndrome neuroléptico maligno, sobretodo al ser un diagnóstico diferencial ante la hipertermia maligna, en los pacientes que utilizan este tipo de fármacos.

\section{Referencias}

1. Jirón M, Machado M, Ruiz I. Consumo de antidepresivos en Chile entre 1992 y 2004. Rev Med Chile 2008 Sep;136(9):1147-54. https:// doi.org/10.4067/5003498872008000900009 PMID:19030659

2. National Center for Health Statistics, Centers for Disease Control and Prevention. Therapeutic drug use. Available at: https:// www.cdc.gov/nchs/fastats/druguse-therapeutic.htm.

3. Attri JP, Bala N, Chatrath V. Psychiatric patient and anaesthesia. Indian J Anaesth. 2012 Jan;56(1):8-13. https://doi. org/10.4103/0019-5049.93337 PMID:22529413

4. Auerbach $A D$, Vittinghoff $E$, Maselli J, Pekow PS, Young JQ, Lindenauer PK. Perioperative use of selective serotonin reuptake inhibitors and risks for adverse outcomes of surgery. JAMA Intern Med. 2013 Jun;173(12):1075-81. https:// doi.org/10.1001/jamainternmed.2013.714 PMID:23699725

5. Caraci F, Leggio GM, Salomone S, Drago F. New drugs in psychiatry: focus on new pharmacological targets. F1000 Res. 2017 Mar;6:397. https:// doi.org/10.12688/f1000research.10233.1 PMID:28408985

6. Park SH, Wackernah RC, Stimmel GL. Serotonin syndrome: is it a reason to avoid the use of tramadol with antidepressants? J Pharm Pract. 2014

Feb;27(1):71-8. https://doi. rg/10.1177/0897190013504957 PMID:24153222

7. Saraghi M, Golden L, Hersh EV. Anesthetic Considerations for Patients on Antidepressant Therapy - Part II. Anesth Prog. 2018;65(1):60-5. https://doi. org/10.2344/anpr-65-01-10 PMID:29509514

8. Teles JS, Fukuda EY, Feder D. Warfarin: pharmacological profile and drug interactions with antidepressants. Einstein (Sao Paulo). 2012 Jan-Mar;10(1):110-5. https://doi.org/10.1590/ S1679-45082012000100024 PMID:23045839

9. Pangalos MN, Schechter LE, Hurko O. Drug development for CNS disorders: strategies for balancing risk and reducing attrition. Nat Rev Drug Discov. 2007 Jul;6(7):521-32. https:// doi.org/10.1038/nrd2094 PMID:17599084

10. Sansone RA, Sansone LA. Serotonin norepinephrine reuptake inhibitors: a pharmacological comparison. Innov Clin Neurosci. 2014 Mar;11(3-4):37-42. PMID:24800132

11. Saraghi M, Golden LR, Hersh EV. Anesthetic Considerations for Patients on Antidepressant Therapy-Part I. Anesth Prog. 2017;64(4):253-61. https://doi. org/10.2344/anpr-64-04-14 PMID:29200376

12. Chen CC, Lin CS, Ko YP, Hung
YC, Lao HC, Hsu YW. Premedication with mirtazapine reduces preoperative anxiety and postoperative nausea and vomiting [table of contents.]. Anesth Analg. 2008 Jan;106(1):10913. https://doi.org/10.1213/01. ane.0000289636.09841.bc PMID:18165563

13. D'Agostino A, English CD, Rey JA. Vortioxetine (brintellix): a new serotonergic antidepressant. P T. 2015 Jan;40(1):36-40. PMID:25628505

14. Thase ME, Mahableshwarkar AR, Dragheim M, Loft H, Vieta E. A meta-analysis of randomized, placebo-controlled trials of vortioxetine for the treatment of major depressive disorder in adults. Eur Neuropsychopharmacol. 2016 Jun;26(6):97993. https://doi.org/10.1016/j. euroneuro.2016.03.007 PMID:27139079

15. Rasmussen KG. Has psychiatry tamed the "ketamine tiger?" Considerations on its use for depression and anxiety. Prog Neuropsychopharmacol Biol Psychiatry. 2016 Jan;64:21824. https://doi.org/10.1016/j. pnpbp.2015.01.002 PMID:25582770

16. Bobo WV, Vande Voort JL, Croarkin PE, Leung JG, Tye SJ, Frye MA. Ketamine for Treatment-Resistant Unipolar and Bipolar Major Depression: Critical Review and Implications for Clinical Practice. Depress Anxiety. 2016 Aug;33(8):698- 
710. https://doi.org/10.1002/ da.22505 PMID:27062450

17. Kennedy SH, Eisfeld BS. Agomelatine and its therapeutic potential in the depressed patient. Neuropsychiatr Dis Treat. 2007 Aug;3(4):423-8. PMID:19300571

18. Kurdi MS, Patel T. The role of melatonin in anaesthesia and critical care. Indian J Anaesth. 2013 Mar;57(2):137-44. https://doi. org/10.4103/0019-5049.111837

PMID:23825812

19. Andersen LP, Rosenberg J, Gögenur I. Perioperative melatonin: not ready for prime time. $\mathrm{Br} J$ Anaesth. 2014 Jan;112(1):7-8. https://doi.org/10.1093/bja/ aet332 PMID:24318695

20. Peck $T$, Wong A, Norman E. Anaesthetic implications of psychoactive drugs. Contin
Educ Anaesth Crit Care Pain. 2010;10(6):177-81. https://doi. org/10.1093/bjaceaccp/mkq037.

21. Aronow WS, Shamliyan TA. Effects of atypical antipsychotic drugs on QT interval in patients with mental disorders. Ann Transl Med. 2018 Apr;6(8):147. https://doi. org/10.21037/atm.2018.03.17 PMID:29862236 